\title{
H I Distribution and Tully-Fisher Distances of Gas-Poor Spiral Galaxies in the Virgo Cluster Region
}

\author{
M. Carmen Toribio and José M. Solanes \\ Departament d'Astronomia i Meteorologia and Institut de Ciències del Cosmos, Universitat \\ de Barcelona. C/ Martí i Franquès, 1; E-08028 Barcelona, Spain \\ mctoribio@am.ub.es, jm.solanes@ub.edu
}

\begin{abstract}
We present aperture synthesis observations in the $21 \mathrm{~cm}$ line of pointings centered on the Virgo Cluster region spirals NGC 4307, NGC 4356, NGC 4411B, and NGC 4492 using the Very Large Array (VLA) radiotelescope in its CS configuration. These galaxies were identified in a previous study of the three-dimensional distribution of $\mathrm{HI}$ emission in the Virgo region as objects with a substantial dearth of atomic gas and Tully-Fisher ( $\mathrm{TF}$ ) distance estimates that located them well outside the main body of the cluster. We have detected two other galaxies located in two of our fields and observed bands, the spiral NGC 4411A and the dwarf spiral VCC 740. We provide detailed information of the gas morphology and kinematics for all these galaxies. Our new data confirm the strong H I deficiency of all the main targets but NGC 4411B, which is found to have a fairly normal neutral gas content. The VLA observations have also been used to discuss the applicability of TF techniques to the five largest spirals we have observed. We conclude that none of them is actually suitable for a TF distance evaluation, whether due to the radical trimming of their neutral hydrogen disks (NGC 4307, NGC 4356, and NGC 4492) or to their nearly face-on orientation (NGC 4411A and $\mathrm{B})$.
\end{abstract}

Subject headings: galaxies: clusters: individual (Virgo) — galaxies: ISM galaxies: kinematics and dynamics — galaxies: spiral — galaxies: structure radio lines: galaxies

\section{Introduction}

The regions around rich clusters are the most obvious sites to evidence the transformation of galaxy disks driven by the surrounding intracluster medium (ICM). The increased 
density of both hot gas and galaxies, as well as the high relative velocities of the latter, set the scene for dramatic effects on their fragile interstellar medium (ISM).

In the local universe, the nearby Virgo Cluster region is an ideal place to quantify these nurturing effects, because its proximity makes it possible to probe the gaseous disks with higher sensitivity and resolution than in any other cluster. Another characteristic that makes this galaxy system very appealing for studies of galaxy evolution is its relative dynamical youth: Virgo has a central region with several substructures in the process of merging, surrounded by suburbia dominated by late-type galaxies that might fall into the cluster during the next Hubble time. As noted by Vollmer et al. (2001) and Solanes et al. (2001), environmental mechanisms such as ram-pressure stripping may see their effectiveness increased during the built-up of clusters.

There is a long list of studies covering a broad stretch of the electromagnetic spectrum that have investigated the impact of cluster residency on the late-type galaxy population. Plenty of them use data from the 21-cm emission line of the abundant, and easy to strip off, neutral hydrogen (HI) of the disks, as the most direct approach to measure the affectation of the ISM. These investigations generally agree in indicating that Virgo spirals, like those inhabiting other rich clusters, tend to have less neutral gas than their field counterparts, and also in finding evidence for a correlation of the H I deficiency with clustercentric distance, with H I-poor disks typically situated close to the cluster cores and galaxies removed from those regions showing normal gas contents (e.g. Havnes \& Giovanelli 1986; Cavatte et al. 1994; Solanes et al. 2001; Gavazzi et al. 2005; Chung et al. 2008). The lack of atomic gas, which usually affects the outer disks, is frequently accompanied by an even more severe truncation of the $\mathrm{H} \alpha$ emission and the corresponding quenching of star formation beyond that truncation radius (e.g. Koopmann \& Kenney 2004; Crowl \& Kennev 2008). Recently, there are also evidences than it could be associated with $\mathrm{H}_{2}$ reduction too (Fumagalli et al. 2009).

Virgo is also the first, and so far the only, cluster region for which the spatial distribution of the H I deficiency has been mapped (Solanes et al. 2002, hereafter Sol02). By using homogenized Tully-Fisher (TF) distance moduli and $21 \mathrm{~cm}$ data from single-dish observations for 161 galaxies, these authors confirmed that the neutral gas deficiency in the Virgo Cluster decreases with increasing 3D barycentric distance. This study, however, also revealed the presence of an unexpectedly large fraction of strongly $\mathrm{H}$ I-deficient spirals with $\mathrm{TF}$ radial distances pointing to a location well outside the cluster body.

Ensuing studies based on both analytic infall models (Sanchis et al. 2002) and $N$-body simulations (Mamon et al. 2004) investigated the possibility that some of the gas-poor spirals in Virgo's suburbia had lost their gas content in a previous passage through the cluster 
core and were now lying near the apocenter of their orbits. Both works lead to the identification by Sanchis et al. (2004) of 13 extremely H I-poor spiral galaxies for which the lack of cold neutral gas could hardly be attributed to ISM-ICM stripping, unless their radial distances were affected by relative errors much larger than the typical uncertainty attributed to TF measurements. Other possibilities for the origin of these H I outliers, such as gas deficiency caused by gravitational interactions (tides or mergers) with companion galaxies, or errors in the H I deficiency estimates arising from morphological misclassifications, were also investigated and considered less probable.

With the aim of shedding more light on this matter, we initiated some time ago a program of dedicated observations of some of the outlying H I-deficient Virgo Cluster spirals found in Sanchis et al. (2004). In this paper, we attempt to improve the results of the aforementioned study, which were based on the analysis of integral galaxy properties retrieved from public databases, by investigating the neutral gas distribution and kinematics, as well as the TF distances, for 4 of these objects by means of deep $21 \mathrm{~cm}$ synthesis observations carried out with the Very Large Array (VLA) in its CS configuration 1 . The paper begins by describing in Section 2 the selection of the targets. The acquisition and reduction of the 21-cm line data, as well as the steps followed in the derivation of the H I synthesis results are discussed in Section 3. In Section 4, we analyze case by case the H I properties of all the galaxies showing $21 \mathrm{~cm}$ emission in the selected fields of view, while in Section 5 we discuss the applicability of the TF technique to the five large spirals that have been observed. Finally, the results and conclusions of this work are given in Section 6 .

\section{Galaxy Selection}

We have used the VLA to observe Virgo Cluster galaxies that are faint in the $21 \mathrm{~cm}$ line. Three of the targets, NGC 4307, NGC 4356, and NGC 4492, belong to the subset of 13 H Ioutliers identified by Sanchis et al. (2004)2. These are galaxies with neutral gas deficiencies deviating by more than $3 \sigma_{\mathrm{DEF}}$ from normalcy. A fourth pointing has been centered on NGC 4411B, another spiral with a less extreme H I deficiency. All these objects are among the most gas-poor spiral galaxies lying on the sky between the M49 subcluster and the W'/W cloud region (see Fig. 1) and have TF estimates of their radial distances suggestive of a possible

\footnotetext{
${ }^{1}$ The VLA is a facility of the National Radio Astronomy Observatory.

${ }^{2}$ Seven other members of this subset are among the targets of the VIVA (VLA Imaging of Virgo in Atomic gas) survey by J. Kenney, J. van Gorkom, and cols. in which the gas is imaged down to a column-density sensitivity of a few times $10^{19} \mathrm{~cm}^{-2}$, similar to that of the present observations.
} 
background location far from the cluster core, provided one adopts the currently preferred Virgo mean distance of $d_{\text {Virgo }} \sim 16-17 \mathrm{Mpc}$, as suggested by both the measurements of $H_{0}$ from HST observations of Cepheids and the spatial distribution of the early type galaxy population and X-ray gas (e.g. Gavazzi et al. 1999; Freedman et al. 2002; Sanchis et al. 2004; Mei et al. 2007). Another characteristic these galaxies have in common is that their systemic velocities do not differ much from the mean cluster velocity. Galaxy properties are compiled in Table1, including a preliminary estimate of their neutral gas deficiency using the following distance-independent calibrator

$$
\mathrm{DEF}=\left\langle\log \bar{\Sigma}_{\mathrm{HI}}(T)\right\rangle-\log \bar{\Sigma}_{\mathrm{HI}}
$$

which compares the logarithms of the expected and observed values of the hybrid H I surface density calculated from the ratio between the intrinsic integrated H I flux and the square of the apparent major optical diameter of a galaxy of morphological type $T$. We have followed Sol02 and adopted for $\left\langle\log \bar{\Sigma}_{\mathrm{HI}_{\mathrm{I}}}(T)\right\rangle$ the values: 0.24 for Sa, Sab types; 0.38 for Sb; 0.40 for Sbc; 0.34 for Sc; and 0.42 for later types in units of $\mathrm{Jy} \mathrm{km} \mathrm{s}^{-1}$ per arcmin square. Thus, taking into account that the rms scatter in DEF for field galaxies is $\sigma_{\mathrm{DEF}}=0.24$, an object with $\mathrm{DEF}>3 \sigma_{\mathrm{DEF}}$ has less than $20 \%$ of the expected H I mass for a galaxy of its morphology.

We have also included in Table 1 four more galaxies located in some of the fields of view of our main targets. These are: VCC 740, a small spiral in the vicinity of NGC 4356; the first component of the pair NGC 4411A/B, very close to its companion galaxy in both projected position and radial velocity (their centers are separated only by $4^{\prime}$ and $11 \mathrm{~km} \mathrm{~s}^{-1}$, respectively), but whose estimated TF radial distance of $\sim 16 \mathrm{Mpc}$ (Sol02) indicates that it is likely a Virgo Cluster member; and two dwarf ellipticals, VCC numbers 933 and 976, lying close to this pair on the sky.

\section{Data Acquisition and Processing}

\subsection{Observations}

The observations published here consist of data obtained at the VLA in its C configuration between July and October 2005. The H I spectral line was observed with the correlator in $4 \mathrm{IF}$ mode using on-line Hanning smoothing.

The observational strategy was designed to achieve the best velocity resolution given the bandwidth needed for each galaxy. For observations where the primary target was an edge-on galaxy (NGC 4307 and NGC 4356), we chose to overlap partially the two IFs, each one with

a bandwidth of $1.526 \mathrm{MHz}$ and a spectral resolution of $24.4 \mathrm{kHz}\left(\sim 5.2 \mathrm{~km} \mathrm{~s}^{-1}\right)$, whereas 
for fields with main targets oriented face-on (NGC 4411B and NGC 4492), the two IFs were centered on the heliocentric velocity of the target, the first with a bandwidth of $1.562 \mathrm{MHz}$ and a spectral resolution of $24.4 \mathrm{kHz}$, and the second using a wider bandwidth of $3.125 \mathrm{MHz}$, but a lower frequency resolution of $97 \mathrm{kHz}\left(\sim 20.8 \mathrm{~km} \mathrm{~s}^{-1}\right)$. The goal was to search for 21 $\mathrm{cm}$ line emission also from possible gaseous tidal tails, extraplanar gas or dwarf companions in the neighborhood of the target objects. The pointing of the field containing NGC 4492 was offset by $3^{\prime}$ towards M49, due to its strong, extended radio continuum emission, in order to avoid systematic effects due to the VLA beam squint as well as to pointing uncertainties in individual VLA antennas (Bhatnagar et al. 2008; Uson \& Cotton 2008).

The July and August observations started in the afternoon. An incidence in the electric system of a substation on August 12th led to the partial loss of the observing time initially allocated for NGC 4492, which was compensated by 3 hours of diurnal observation on September 18th. The NGC 4411B field was also observed during the daytime on the 1st and 2 nd of October ( 5 and $4 \mathrm{hrs}$, respectively). Solar interference was therefore significant only for the September and October observations.

Each galaxy was observed for about 6 hours with an overhead of $\sim 2$ hours for calibration which included two 10-minute scans on each day on the primary calibrator $3 \mathrm{C} 286=1331+305$ (J2000). The rest of the observing sequences consisted of 30-minute scans of the target fields interspersed with 10-minute observations of the corresponding secondary calibrator. For the data acquired between July and September, 3C273 = 1229+020 (J2000), with a flux of $\sim 32$ Jy, was used as a secondary phase and bandpass calibrator. This calibrator was too close to the Sun during our October observations of the NGC 4411B field. We therefore modified our strategy and observed J1254+116 as a secondary calibrator. A summary of the observing parameters is provided in Table 2 .

\subsection{Data Calibration}

The raw UV data were reduced using the Astronomical Image Processing System (AIPS) software package distributed by the National Radio Astronomy Observatory 3

The observations of NGC 4307, NGC 4356, and NGC 4492 were calibrated in a similar way. First, we discarded corrupted data by inspecting a pseudo-continuum database obtained from the vector average of visibilities for channels 4 to 60 at each time stamp - the remaining channels in the low and high velocity ends of the bandpass were discarded from the beginning.

\footnotetext{
${ }^{3}$ Unless otherwise stated, all the quoted routines in capital letters belong to this package.
} 
The primary flux calibrator 3C286 was used then to determine an initial bandpass as well as zeroth-order amplitude and phase calibration. Next, pseudo-continuum images of 3C273 were obtained and subsequently used to calculate a secondary phase calibration. The same source was the basis to determine a secondary bandpass calibration. This calibration was applied to the data on the observing fields by linear interpolation of bandpass and global phases. Finally, phase self-calibration of the data on the galaxies was applied, and for NGC 4307 and NGC 4356, the two IFs were joined by means of the task UJOIN (channel-by-channel visibility averages for the overlapping channels; see Matthews \& Uson 2008). Regarding the NGC 4492 field, we note that during the make-up observation scheduled on September 18th we acquired 3 hours of data that suffered from solar interference. This forced us to reject baselines shorter than $1 \mathrm{k} \lambda$ for that day.

The reduction process just described was unsuitable to the observations of the NGC 4411B field. After discarding corrupted data, we calculated amplitude and phase calibrations from the observations of $3 \mathrm{C} 286$ and $1254+116$. A solution for the shape of the bandpass obtained using 3C286 was applied to the whole data at the same time that the amplitude and phase solution, which was interpolated for NGC 4411B dataset by means of simple linear connection between phases. As for the NGC 4492 September data, we discarded spacings less than $1 \mathrm{k} \lambda$ to avoid the strong contamination by solar RFI.

\subsection{Continuum Emission Subtraction}

Continuum emission in our fields was estimated by imaging the vector average of visibilities for line-free channels and subsequently subtracted from the datacube with UVSUB.

We note that comparison of our flux measurements of the brightest sources in each field with the corresponding flux values listed in the NRAO VLA Sky Survey (NVSS, Condon et al. 1998) shows good agreement, although, on average, our flux measurements are $\sim 5 \pm 2 \%$ larger than the NVSS values. Given the low signal-to-noise ratio $(\mathrm{S} / \mathrm{N})$ of the spectral signal of our targets, this does not affect our total H I flux estimates. These discrepancies, however, were accounted for in the calculation of the corresponding uncertainties.

As mentioned on $\S 3.2$, for targets close to the Sun, the images were obtained after excluding the baselines most affected by solar contamination. However, we could not eliminate solar RFI completely, which prompted us to make a second continuum subtraction. The routine UVLIN was used to fit and subtract a first order polynomial to the real and imaginary components of each visibility through the line-free channels. Following the recommendation by Cornwell. Uson, \& Haddad (1992), we applied UVLIN also to the data not affected by 
solar contamination in order to subtract any residual continuum emission. Subsequently, we obtained the channel images and examined the statistics of the image cubes to check for artifacts and, in particular, to verify that the distribution of noise in our data cubes was Gaussian-like. Finally, we proceeded to concatenate the datasets for those objects with observations split in two different dates (NGC 4492 and NGC 4411B).

\subsection{H I Synthesis Results}

\subsubsection{Channel Maps}

Image cubes were constructed for the NGC 4307, NGC 4356, and NGC 4411B fields using robustness parameters $\Re=-1$ (closer to uniform weighting) and $\Re=0.7$ (closer to natural weighting). For all galaxies but NGC 4492, we present the results for $\Re=0.7$

as it provides the best compromise between the $\mathrm{S} / \mathrm{N}$ and resolution when data have full UV-coverage (Briggs 1995).

The low S/N of the data on NGC 4492 forced us to smooth and taper the observations to improve our sensitivity. Channel maps were obtained from IF 2 (spectral resolution $\sim 20.8$ $\mathrm{km} \mathrm{s}^{-1}$ ) by using a robustness parameter $\Re=1$, which results in lower noise levels and a wider, but still acceptable, synthesized beam size. A Gaussian taper (with a $9 \mathrm{k} \lambda$-width at the $30 \%$ level both in the $\mathrm{U}$ and $\mathrm{V}$ directions) additional weighting was applied on the visibilities to lower the contribution of the long-baseline datapoints.

In all cases, the channel images were CLEANed and the clean components restored with a Gaussian beam similar to the synthesized beam. The characteristics of the deconvolved images are summarized in Table 3 ,

\subsubsection{Moments}

The cleaned image cubes were used to calculate total H I flux images, as well as first and second velocity moments of the $21 \mathrm{~cm}$ emission.

We decided whether to keep or not a pixel in the integration by examining a spatially and frequency smoothed version of the datacubes. The spatial smoothing was done by convolving with a Gaussian kernel, whereas a Hanning smoothing was applied in velocity. We selected those pixels from the original datacubes that were above the $3 \sigma$ level in the smoothed counterpart, except for NGC 4492, for which the $2 \sigma$ level was used (otherwise, almost no signal was left from the galaxy). All flux with absolute value above $0.5 \sigma$ was 
integrated along the velocity axis to obtain the total H I map, and intensity-weighted first and second order moments were computed. Finally, the H I intensity map was corrected for primary beam attenuation and scaled to the column density $N_{\mathrm{HI}}$ of the gas, assuming optically thin H I. When estimating the errors in the latter map, we scaled the rms noise in the channel maps to the square-root of the mean number of adjacent channels that contributed to the total intensity image, and then added a $5 \%$ independent uncertainty arising from calibration and correction for primary beam attenuation.

The diameter of the $\mathrm{H}$ I disks was measured at a column density of $10^{20}$ atoms $\mathrm{cm}^{-2}$ (no correction for beam smearing was applied). The reported uncertainties take into account variations in position angle of major axis as well as the correlation introduced by the synthesized beam (Table 4).

\subsubsection{Global H I Profiles}

In order to determine if we have recovered all of the flux from single-dish measurements, simulated line profiles were derived. The latter were calculated by integrating over the spatial axes for each channel the primary-beam attenuation-corrected emission used in the moment calculation ( $\S$ 3.4.2) .

We have measured $W_{20}$ and $W_{50}$, the profile width at the $20 \%$ and $50 \%$ of the peak intensity, respectively. For those cases in which a clear double-peaked profile is found (VCC 740, NGC 4411A and NGC 4411B), the peak fluxes on both sides were considered separately when calculating the linewidths. In the remaining cases we used the overall peak flux. The H I linewidths were subsequently corrected for broadening effects due to the finite spectral resolution of the instrument by following the considerations of Verheijen \& Sancisi (2001). The adopted broadening corrections for the cubes with a spectral resolution of $5.2 \mathrm{~km} \mathrm{~s}^{-1}$ were $\delta W_{20}=0.86 \mathrm{~km} \mathrm{~s}^{-1}$ and $\delta W_{50}=0.56 \mathrm{~km} \mathrm{~s}^{-1}$, whereas when the resolution was 20.8 $\mathrm{km} \mathrm{s}^{-1}$, we adopted $\delta W_{20}=12.0 \mathrm{~km} \mathrm{~s}^{-1}$ and $\delta W_{50}=7.88 \mathrm{~km} \mathrm{~s}^{-1}$.

The heliocentric systemic velocity, $v_{\text {sys }}$, was derived as the average of velocities of channels at 20 and $50 \%$ of the peak flux of the profile, and the total H I flux was obtained by integrating the $21 \mathrm{~cm}$ line profiles along the velocity axis.

Since some of the profiles have low $\mathrm{S} / \mathrm{N}$, we decided to measure the kinematic parameters defined above by running Monte Carlo simulations for each one of the profiles. The values quoted in Table 4 correspond to the mean and 1- $\sigma$ deviation of the distribution of measurements from one thousand random realizations of the profiles by taking into account the flux in each channel and its rms error. The latter was estimated from the rms noise in 
the channel maps and the correlation introduced by the synthesized beam. The quoted error in the total H I flux has been estimated by adding in quadrature the error estimates from the Monte Carlo technique and a 5\% uncertainty arising from the calibration and the correction for primary beam attenuation.

We find, in general, good agreement with single-dish observations (Table 4). Especially remarkable is the close match between the shapes, linewidths, and total flux densities of our VLA H I line profiles and their counterparts in the ongoing Arecibo Legacy Fast ALFA (ALFALFA) extragalactic H I survey (Giovanelli et al. 2005), which recently released the results for the strip of the Virgo Cluster region where our targets are located (Kent et al. 2008).

\subsubsection{Position-Velocity Diagrams and Rotation Curves}

Position-velocity (PV) diagrams along the major axis of each target were also obtained by taking slices of the data cubes through the optical centers and estimating their position angle from the moment maps or from the rotation curves in those cases where this was possible.

Major-axis velocities as a function of angular radius were exclusively derived for the two components of the pair NGC 4411A/B and the dwarf spiral VCC 740, since these were the only targets detected with a high $\mathrm{S} / \mathrm{N}$ (see next section). To infer the rotation curves we

have fitted both a Brandt model and the standard iterative tilted-ring algorithm (Begeman 1989).

\section{Case by Case Analysis of the H I Synthesis Data}

Table 4 summarizes the results inferred from our analysis of the VLA observations for all the galaxies detected. In the printed version of the manuscript we include a complete graphical layout of the NGC 4307 field (Figs. 2 3). Images for the rest of our VLA pointings are available in the electronic version of the manuscript.

We now comment on the properties of the target galaxies from our VLA observations. 


\subsection{NGC 4307}

The H I gas disk of NGC 4307 has very small dimensions and is found only deep within the optical disk, in agreement with the strong gas-deficiency estimated for this galaxy. The deficiency parameter DEF (eq. [1]) measured from our VLA flux (see Table 4) shows a pretty good consistency with the value of 1.41 one can infer from the observed $\mathrm{H}$ I flux provided by (Kent et al. 2008). We remark that this new value is also in agreement with the extremely H I-deficient status reported in previous works (e.g. Sol02; Gavazzi et al. 2005).

The 0th-order moment map shows that the maximum of the $21 \mathrm{~cm}$ emission is displaced $\mathrm{SE}$ with respect to the optical center, in a direction nearly perpendicular to the major axis, with an excess of emission at the approaching side. This asymmetry shows up also in the global H I profile, which has a peaked appearance indicative of centrally concentrated gas. The estimated offset of $\sim 7^{\prime \prime}$ is less than half of both the synthesized beam size and the mismatch expected to arise from edge-on ram-pressure stripping in hydrodynamic simulations (Kronberger et al. 2008).

There are no other pieces of evidence susceptible to being interpreted as environmental effects down to the sensitivity limit of the measurements. Moreover, the galaxy is too inclined to allow one to disentangle whether the observed asymmetries can be ascribed to noncircular motions associated with a spiral arm.

The PV diagram along the major axis is still rising at the edge of the measured H I distribution, as if the gas was rotating almost as a pure solid body. Our VLA data is compatible to a large extent with the $\mathrm{H} \alpha$ rotation curve derived by Gavazzi et al. (1999), which shows a hint of a turn over on both sides of the galaxy. We discuss in Section 5 the risks of using extremely $\mathrm{H}$ I-deficient galaxies like this one in a TF analysis.

\subsection{NGC 4356 and VCC 740}

The properties of the neutral hydrogen in NGC 4356 resemble those of NGC 4307. This is a nearly edge-on galaxy that like the former one, and even more strongly so, has a very small gas distribution compared with its optical dimensions. The PV diagram along the major axis also rises steeply and shows no signs of turning over, as in the H $\alpha$ rotation curve measured by Gavazzi et al. (1999). There is similarly a misalignment between the distribution and motion of the atomic gas and the optical disk. As in the former case, this offset is less than half the synthesized beam size and, hence, relatively small and not necessarily indicative of ram-pressure pushing. 
For this galaxy, our VLA linewidth and flux values are somewhat smaller that the most recent estimates by Kent et al. (2008). Given the low S/N of this galaxy, the observed difference in total fluxes, which is only of tenths of a mJy $\mathrm{km} \mathrm{s}^{-1}$, does not necessarily imply that some flux has been lost due to the missing short baselines in our synthesis aperture images. Instead, the discrepancies can be the result of low-baseline ripples that seem to affect the single-dish profile.

VCC 740, another highly inclined galaxy detected in this same pointing, has, in contrast, quite a strong $\mathrm{S} / \mathrm{N}$ and a total $\mathrm{H}$ I flux that is about the same that in NGC 4307. Its H I map suggests that the neutral gas has a sharp cutoff at the optical radius of the disk on the approaching side, while the other side has a more gradual falloff and is somewhat more extended. In spite of giving the impression that part of the gas might be missing, both ours and the ALFALFA flux measurements actually result in a negative deficiency parameter DEF of $\sim-0.2$, indicative of a perfectly normal H I content.

The gas velocity field of VCC 740, on the other hand, exhibits some warping, as well as inner contours parallel to the minor axis, indicating that $V_{\text {rot }}(R) \propto R$, as the PV diagram shows. Further out on the SE side, the contours show the classical $V$-shape, suggesting that at the sensitivity limit of the measurements the rotation speed is just about to become nearly constant. In the rotation curve model fits, the warp of the velocity field increases the position angle on the external part of the disk. Both Brandt and tilted-ring models yield an estimated inclination of $\sim 70^{\circ}$ for the internal disk region that drops to $\sim 45^{\circ}$ on the outside. The fact that the velocity field of this galaxy shows a clear rotation pattern reinforces its morphological classification as a dwarf barred disk instead of the IB type assigned in the LEDA. The angular resolution of our VLA measurements, however, is insufficient to observe the effects of the bar on the gas velocities. No signs of interaction are found between VCC 740 and NGC 4356.

\subsection{NGC 4411A/B, VCC 933 and VCC 976}

Contrarily to what has happened with NGC 4307 and NGC 4356, the two low surface brightness spirals NGC 4411A and B have produced integrated H I fluxes that are inconsistent with the values quoted in Sol02, which were estimated in earliest mapping attempts done with the Arecibo antenna (Havnes 1981). Discrepancies with this and other old single-dish measurements (e.g. Hoffman et al. 1989) are ascribed, however, solely to the amplitudes, as the shapes of the global H I profiles look very similar.

Our new data, reinforced by the newest single-dish measurements done at Arecibo by 
the ALFALFA team (Kent et al. 2008), imply that NGC 4411A and NGC 4411B loose their initial status of objects with moderate and strong H I deficiency, respectively (see Table 1), to be both reclassified as galaxies with quite a normal gas abundance, in accordance with the visual impression obtained from the H I contours overlaid on the Digitized Sky Survey (DSS) image.

These maps show that these two galaxies have H I distributions extending beyond their optical disks, which for NGC 4411A reach up to nearly twice the optical radius, except on the NE side where the H I contours appear compressed. In this latter galaxy the H I is concentrated in a ring with two important regions of emission that emanate perpendicularly from the ends of the bar. The 0th-order map of NGC 4411B shows an even wider major ring-like structure with a noticeable excess of emission at its northern half near the outer edge. We do not find important displacements from the optical disks, the most remarkable feature being the presence of a depression in the center of both galaxies, as found in other LSB galaxies (de Blok, McGaugh, \& van der Hulst 1996).

We fail in detecting neither gas bridges nor significant intergalactic H I signals between these two targets. Given that both galaxies show normal disk emission with only mild alterations of the symmetry, comparable to those seen in more isolated objects (Kornreich et al. 2000), we feel compelled to classify the system NGC 4411A/B as a visual pair (see also $\S 5$ ).

Regarding the kinematics of the neutral gas, we note that the PV diagram of NGC 4411B shows a steep rise well within the stellar disk (of small amplitude given its near faceon orientation) followed by a sharp bend towards a flat part, — with indications of a modest decline on the receding side at the largest radii where the $\mathrm{HI}$ is detected-, consistent with the shape of the radial velocity contours and with the well behaved double-horned global profile. In NGC 4411A, the turnover in the rotation velocity is not complete and the $21 \mathrm{~cm}$ line profile is affected by a larger asymmetry. The observed behavior of the PV diagrams is typical of objects with a compact distribution of their luminous matter.

For these two galaxies we have gone a step further and inferred also major-axis rotation velocities with the aim of estimating the orientations of the gaseous disks. The apparent inclination inferred from the tilted-ring technique for NGC 4411A is $29_{-3.7}^{+5.2}$ degrees, while for NGC $4411 B$ we get $i=26_{-4.7}^{+4.4}$ degrees. Brandt curve fits to the whole velocity fields result in similar inclinations of $\sim 27^{\circ}$ and $\sim 28^{\circ}$, respectively. Yet it shouldn't be forgotten that the bumps in the H I line data associated with spiral arms and, especially, the near face-on orientation of these disks, make rotation curve model fits to the velocity fields uncertain and, caveat lector, unable of giving a precise inclination angle. In the next section, we discuss the problems arising from the derivation of this parameter in low-inclination galaxies and provide alternative estimates based on optical images. 
Our VLA observations have not detected $21 \mathrm{~cm}$ line emission coming from any of the two dwarf elliptical galaxies VCC 933 and VCC 976 also present in this field.

\subsection{NGC 4492}

In spite of the strong image-degrading effects of the Sun for the second half of the observations of NGC 4492, we succeed in detecting its H I signal at a quality level comparable with the previous single-dish observations carried out by Haynes \& Giovanelli (1986) and Hoffman et al. (1989) - and not too different from the one achieved by the ALFALFA measurements, which have a $\mathrm{S} / \mathrm{N}$ of 4.6 .

In this galaxy, the detected neutral hydrogen is located within the optical radius and shows an important elongation in the SE-NW direction, almost perpendicularly to the galaxy major axis. The H I distribution is strongly asymmetric, with the peak of the $\mathrm{HI}$ emission shifted some $30^{\prime \prime}$ to the East from the optical center of the galaxy. This gives rise to a synthesized line profile with decreasing flux toward the approaching side. By integrating the latter, we find a total H I flux about a 30\% smaller than the ALFALFA's value (see Table 4). Again, one may wonder whether possible flux losses in our estimation arising from too a strict rejection of the short baselines could explain this difference. In this respect, we note that during the application of the Gaussian UV-taper weighting to obtain the final datacubes some testing done varying the width of the tapering function showed that the resulting total $\mathrm{H}$ I flux was not significantly affected. Therefore, given the low $\mathrm{S} / \mathrm{R}$ of the detections, the discrepancies in total flux and linewidths with respect to singledish measurements could have been originated by low-level baseline ripples that seem to affect the ALFALFA profile. With this caveat in mind, our VLA measurements assign a new H I deficiency parameter of 1.21 to NGC 4492, which indicates that the gas content for this galaxy could be less than $6 \%$ of the expectation value.

The asymmetries on the spatial distribution of the gas for this galaxy are reproduced in the H I dynamics. Thus, the velocity map of NGC 4492 reveals a possible displacement of the dynamical center from the light center (consistent with that observed in the 0th-order map), while on the PV diagram along the major axis most of the emission is found coming from the receding side. The low $\mathrm{S} / \mathrm{N}$ of the datacubes, the small size of the $\mathrm{H}$ I disk with respect to the beam size, as well as a not too favorable orientation of this galaxy, prevent any attempt to fit a rotation curve model to the velocity data. 


\section{TF-based Distance Estimates to our Galaxies}

The most striking aspect of the Virgo's galaxies selected in the present study is not their high deficiency of neutral gas, but the possibility that this has been attained outside the cluster environment. While in the outskirts of clusters gas stripping can happen by galaxygalaxy interactions in infalling groups or through collisions with lumps of intergalactic gas (see, for instance, Crowl \& Kenney 2006, 2008), strong H I deficiencies in the periphery of clusters are expected to be an exception to the rule.

The classification by Sanchis et al. (2004) of a spiral galaxy as an H I outlier relied on its TF distance. The latter was inferred from the disk's maximum rotation speed $V_{\text {rot }}$, — which is expected to be a proxy to the total mass of the galaxy and, therefore, also to its intrinsic luminosity - , measured via the width of the H I spectral line. It is then of fundamental importance to assess the feasibility of this technique in galaxies like ours which have gaseous disks deeply altered.

From Guhathakurta et al. (1988) to Cortés, Kenney, \& Hardy (2008) the literature is full of TF studies highlighting the risks of using the gas kinematics in the determination of distances to H I-deficient galaxies. The most straight reasoning being that truncated gas disk measurements could underestimate the rotation velocity of a galaxy and, therefore, its mass, biasing low the derived radial distance. However, depending on the interaction mechanism and its geometry, the $\mathrm{H}$ I that does not get dislocated may also lost temporarily its equilibrium within the global galactic potential - externally induced disturbances on the kinematics of disks are erased in about 1 Gyr (e.g. Dale et al. 2001), a time during which two interacting galaxies can move hundreds of kiloparsecs apart. These effects, as well as induced noncircular or nonplanar gas motions, which may even lead to an overestimation of the true $V_{\text {rot }}$ (Kronberger et al. 2007), possible changes in the observed luminosity resulting from alterations in the star formation rate or, simply, the fact that even for undisturbed galaxies in many cases there is evidence of noncircular motions in their central regions (for

instance, due to bars; Valenzuela et al. 2007), could make the application of the TF technique in strong H I-deficient galaxies totally inefficient.

All this drives us to regard critically the distance estimates of the three galaxies in our sample that exhibit severely truncated gas disks: NGC 4307, NGC 4356, and NGC 4492. Certainly, we have not found evidence of a recent gravitational encounter in our $21 \mathrm{~cm}$ line imaging data in the form of gaseous tails and bridges for any of them. Nor the closeness of the systemic velocity of the first two galaxies to the mean cluster velocity supports a recent ram-pressure event inside the core. Yet the fact we do not detect a flat part in their rotation curves, as well as the irregularities in the spatial distribution and kinematics of the H I evidenced in the VLA maps of these objects do not allow us to state confidently that 
the dynamical equilibrium of the neutral gas has been fully restored after the removal event. Neither can we assert that their luminosities have not been affected. Therefore, we believe that it is not legitimate to use the TF technique to derive the radial distance to any of these three galaxies and that, consequently, their published TF distance measurements might well be largely in error.

Compared to the previous objects, NGC 4411B and its close neighbor NGC 4411A exhibit regular and symmetric gas velocity fields, with flat extended outer parts that appear to satisfy the basic tenet that underlies a TF study. In this case, however, the attempts of estimating the radial distance to these two galaxies are thwarted for an unfavorable viewing angle. At low apparent inclinations $\left(i \lesssim 40-45^{\circ}\right)$, estimates of the orientation of disks become more uncertain, led to deprojected quantities with divergent errors as a face-on orientation is approached, and are skewed towards larger values by nonaxysimmetric features in the images (see Andersen \& Bershady 2003 and references therein). As a result, the total fractional error in the radial distance for low-inclination galaxies largely exceeds $15 \%$, a value usually adopted as representative of the typical uncertainty in the distance arising from good-quality TF data.

This situation is illustrated in Figure 4, where we show the radial distances to NGC 4411A and $\mathrm{B}$ reported in some of the TF catalogs used by Sol02. Our synthesized H I line profiles have been used to estimate the intrinsic rotational velocities, which we have calculated by exactly following the same prescriptions adopted in the referenced studies that in some cases apply non-null turbulent motion corrections. The uncertainty resulting from inclination measures is shown by a vertical bar whose extent is set by the most extreme values of this angle ever assigned to our galaxies in the literature (which range from $\sim 20^{\circ}$ to $\left.\sim 55^{\circ}\right)$. Open diamonds in the plots indicate radial distances published in the cited references. It is obvious from this figure that the inability to infer accurate inclinations prevents us from establishing the location of both galaxies along the LOS within the entire Virgo Cluster region.

We have also depicted in Figure 4 the H I-based radial distances inferred from our own measurements of the inclination of these galaxies (Sec. 4.3). Double pointed arrows encompass the ranges of distances corresponding to the values of inclinations and errors derived from inspection of the residuals of our tilted-ring model fit to the H I velocity fields. The well-known limitations of the weighting scheme included in the modeling of the H I rotation curves when it comes to measuring inclinations below $40^{\circ}$ Andersen \& Bershadv 2003), have led us to make as well independent fits to the orientation parameters of these disks from Sloan Digital Sky Survey (SDSS) images. We have used the package GALFIT (Peng et al. 2002) to decompose the r-band images of both galaxies into several components. 
The sky contribution apart, we have fitted a bulge plus an exponential disk to the image of NGC 4411B, while for NGC 4411A a bar component has been added too. We have followed Binney \& de Vaucouleurs (1981) and adopted intrinsic axis ratios of 0.18 for NGC 4411A $(\mathrm{SBc})$ and of 0.13 for NGC 4411B (Scd). All this gives estimated inclinations of about $24^{\circ}$ for the stellar disk of NGC 4411A and of $\sim 16^{\circ}$ for the one of NGC 4411B. The uncertainties in these values are difficult to determine, as the analysis of the optical images involves a large number of parameters. Resulting distances are indicated in the plots by asterisks. Comparison with the results from the H I shows that for nearly face-on disks in the Virgo Cluster region inclinations differing by $\sim 10^{\circ}$ can lead, depending on the TF relationship adopted, to differences in distance exceeding $10 \mathrm{Mpc}$. The very uncertain radial distances and the lack of unmistakable signs of an ongoing interaction do not make it possible to assert that these galaxies are physically connected in spite of their proximity in $z$-space.

Another feature of Figure 4 that draws one's attention is the existence of considerable author to author fluctuations in the estimated TF radial distances that cannot be just ascribed to the uncertainties in the observational parameters entering this relationship. For highly inclined galaxies, such discrepancies can be traced back mostly to the adopted TF template, which for a given passband can show systematic variations among different authors, even when similar samples of calibrators are used. We note, for instance, that small datasets are rather sensitive to the trimming of the data and, hence, to the, somewhat subjective, identification of the most deviant measurements. Besides, differences on the fitting methods, or on the adopted data weighting, as well as morphological and incompleteness biases (see, for instance, Giovanelli et al. 1997), may give raise to significant variations in the slope and zero-point coefficients of the TF relation. As shown in Figure 5, where we compare the $B$ band TF template relations defined in the studies of the Virgo Cluster considered by Sol02, the absolute magnitude assigned to a given galaxy, regardless of its inclination, can vary up to $\sim 1.5 \mathrm{mag}$ depending on the calibration adopted. This exceeds by far the typical error of $\sim 0.1-0.2$ mag usually assigned to the zero-point calibration of individual TF templates.

\section{Summary and Conclusions}

This is the first $21 \mathrm{~cm}$ synthesis survey of spiral galaxies ever made in which the targets have been specifically chosen on the basis of their expected dearth of cold gas. It has been motivated by the detection, in previous investigations of the neutral gas content on spirals in the Virgo Cluster region, of a significant number of severely H I-deficient disks supposedly located, according to their TF distance estimates, beyond the maximum rebound radius

galaxies can bounce after infall. According to this location, these galaxies could not owe 
their H I deficiency to interactions within the cluster environment.

Our high-sensitivity VLA observations have been aimed at characterizing in detail the spatial distribution and kinematics of the neutral gas in four galaxies suspected of being $\mathrm{HI}$ I-outliers, in a first effort to gain a better understanding of the origin of this class of objects. At the same time, these synthesis observations in the $21 \mathrm{~cm}$ line have provided direct evidence of the risks involved in the application of the TF relationship to disturbed or nearly face-on disks, which can render the derived distances unreliable.

We have detected a total of six galaxies within the four fields initially selected. The main conclusions of the analysis of our VLA data are as follows:

1) We confirm the strong H I deficiency of three of our four main targets, NGC 4307, NGC 4356, and NGC 4492 (inferred H I contents are a factor $\gtrsim 20$ lower than their corresponding standard values), which is pronounced through the reduced extension of the gaseous disks, a characteristic typical of ram-pressure-stripped galaxies. In contrast, we find that the integrated H I fluxes of our fourth target, NGC 4411B, and its companion, NGC 4411A, are $\sim 2-3$ times larger that the old single-dish values used to estimate their $\mathrm{HI}$ deficiency in Sol02. Our measured VLA fluxes, - which for all our targets are compatible with those inferred from the new, sensitive ALFALFA extragalactic H I survey-, indicate that the H I contents of these last two galaxies deviate less than $1 \sigma$ from normalcy. This is consistent with our observation that their $\mathrm{H}$ I disks extend beyond their optical counterparts, so that only the outermost portions of the cold gas distributions have been affected, if at all. A sixth galaxy with a healthy amount of cold gas, the dwarf spiral VCC 740 , has been detected in the field of NGC 4356.

2) Visual inspection of the images of the most gas-deficient galaxies has revealed signs of asymmetries and lopsidedness, as well as small offsets of the dynamical centers with respect to the optical ones. These are all suggestive, albeit not conclusive, indications of possible gravitational interactions and/or ram-pressure effects, as deviations from flat, axisymmetric disks are also known to prosper in isolated galaxies. This, and the fact that we have not found evidence of gaseous tails or bridges within the limit we have been able to trace the H I $\left(\sim 3-5 \times 10^{19} \mathrm{~cm}^{-2}\right.$ channel $^{-1}$ at the $3 \sigma$ level $)$ appear to indicate that none of the galaxies investigated has undergone recent gravitational interactions. This means, in particular, that our VLA observations reinforce the classification of NGC 4411A/B as a virtual pair in spite of their closeness on the observational space phase.

3) Our three targets with highly truncated gas disks exhibit rotation velocities that are still rising at the last measured points. Moreover, the observational evidence gathered do not allow us to assert with complete confidence that the gas remaining tied to the disks has 
regain dynamical equilibrium, nor the extent to which the luminosity of these H I-deficient galaxies could have been affected. The classification of these objects as H I-outliers could therefore simply obey to the inefficient estimate of their radial distances by means of the TF relationship. The fourth target, NGC 4411B, as well as it space phase neighbor NGC 4411A, show, in contrast, extended gas disks with regular and symmetric velocity fields. In spite of being galaxies presumably in virial equilibrium, their TF-based distances are also problematic because of their nearly face-on apparent orientation, which results in the inability to determine accurate inclinations. This translates into a considerable uncertainty - larger than the resolution necessary to determine unambiguously the region (infall or cluster) where a galaxy belongs - when it comes to placing these two galaxies along the LOS within the Virgo Cluster region.

Aperture synthesis observations in the $21 \mathrm{~cm}$ line like the ones presented here are fundamental for probing the impact of cluster residency on the spiral population. Further insight into the identification of the physical processes disturbing the disks can be gained by supplementing this type of data with multifrequency observations.

This work was partly supported by the Dirección General de Investigación Científica y Técnica, under contracts AYA2006-01213 and AYA2007-60366. M.C.T. acknowledges sup-

port from a fellowship of the Ministerio de Educación y Ciencia of Spain. We are grateful to all the people and institutions that have made possible the LEDA (http://leda.univ-lyon1.fr/), GOLDMine (http://goldmine.mib.infn.it/), DSS (http://archive.stsci.edu/cgi-bin/dss_form), and SDSS (http://cas.sdss.org) databases.

\section{REFERENCES}

Andersen, D.R., \& Bershady, M.A. 2003, ApJ, 599, L79

Begeman, K.G. 1989, A\&A, 223, 47

Binney, J., \& de Vaucouleurs, G. 1981, MNRAS, 194, 679

Böhringer, H., Briel, U.G., Schwarz, R.A., Voges, W., Hartner, G., \& Trumper, J. 1994, Nature, 368, 828

Bhatnagar, S., Cornwell, T.J., Golap, K., \& Uson, J.M. 2008, A\&A, 487, 419

Briggs, D.S. 1995, High Fidelity Deconvolution of Moderately Resolved Sources, Ph.D. thesis, New Mexico Institute of Mining and Technology. Available at http://www.aoc.nrao.edu/ftp/dissertations/dbriggs/diss.html 
Cayatte, V., Kotanyi, C., Balkowski, C., \& van Gorkom, J.H. 1994, AJ, 107, 1003

Cayatte, V., van Gorkom, J.H., Balkowski, C., \& Kotanyi, C. 1990, AJ, 100, 604

Chung, A., van Gorkom J.H., Kenney, J.D.P., Crowl, H., Vollmer, B., \& Schiminovich, D. 2008, in 'Formation and Evolution of Galaxy Disks', ed. J.G. Funes, et al., ASP Conference Series, 396, 127

Condon, J.J., Cotton, W.D., Greisen, E.W., Yin, Q.F., Perley, R.A., Taylor, G.B., \& Broderick, J.J. 1998, AJ, 115, 1693

Cornwell, T., Uson, J.M., \& Haddad, N. 1992, A\&A, 258, 583

Cortés, J.R., Kenney, J.D.P., \& Hardy, E. 2008, ApJ, 683, 78

Crowl, H.H., \& Kenney, J.D.P. 2006, ApJ, 649, L75

Crowl, H.H., \& Kenney, J.D.P. 2008, AJ, 136, 1623

Dale, D.A., Giovanelli, R., Haynes, M.P., Hardy, E., \& Campusano, L.E. 2001, AJ, 121, 1886

de Blok, W.J.G., McGaugh, S.S., \& van der Hulst, J.M. 1996, MNRAS, 283, 18

Ekholm T., Lanoix, P., Teerikorpi, P., Fouqué, P., \& Paturel, G. 2000, A\&A, 355, 835 (Ekh00)

Federspiel, M., Tammann, G.A., \& Sandage, A. 1998, ApJ, 495, 115 (FTS98)

Fouqué, P., Bottinelli, L., Gouguenheim, L., \& Paturel, G. 1990, ApJ, 349, 1 (Fou90)

Freedman, W.L., et al. 2002, Nature, 371, 757

Fumagalli, M., Krumholz, M.R., Prochaska, J.X., Gavazzi, G., \& Boselli, A. 2009, ApJ, 697, 1811

Gavazzi, G., Boselli, A., Scodeggio, M., Pierini, D., \& Belsole, E. 1999, MNRAS, 304, 595 (Gav99)

Gavazzi, G., Boselli, A., van Driel, W., \& and O’Neil, K. 2005, A\&A, 429, 439

Giovanelli, R., \& Haynes, M.P. 1985, ApJ, 292, 404

Giovanelli, R., Avera, E., \& Karachentsev, I.D. 1997, AJ, 114, 122

Giovanelli, R., Haynes, M.P., Herter, T., Vogt, N.P., Da Costa, L.N., Freudling, W., Salzer, J.J., \& Wegner, G. 1997, AJ, 113, 53 
Giovanelli, R., Haynes, M.P., Kent, B.R., et al. (The ALFALFA Collaboration) 2005, AJ, 130, 2598

Guhathakurta, P., van Gorkom, J.H., Kotanyi, C.G., \& Balkowski, C. 1988, AJ, 96, 851

Haynes, M.P. 1981, AJ, 86, 1126

Haynes, M.P., \& Giovanelli, R. 1986, ApJ, 306, 466

Hoffman, G.L., Glosson, J., Helou, G., Salpeter, E.E., \& Sandage, A. 1987, ApJS, 63, 247

Hoffman, G.L., Williams, B.M., Lewis, B.M., Helou, G., \& Salpeter, E.E. 1989, ApJS, 69, 65

Irwin, J.A. 1994, ApJ, 429, 618

Kent, B.R., Giovanelli, R., Haynes, M.P., Martin, A.M., Saintonge, A., Stierwalt, S., Balonek, T.J., Brosch, N., \& Koopmann, R.A. 2008, AJ, 136, 713

Koopmann, R.A., \& Kenney, J.D.P. 2004, ApJ, 613, 866

Kornreich, D.A., Haynes, M.P., Lovelace, R.V.E., \& van Zee, L. 2000, AJ, 120, 139

Kraan-Korteweg, R.C., Cameron, L.M., \& Tammann, G.A. 1988, ApJ, 331, 620 (KCT88)

Kronberger, T., Kapferer, W., Schindler, S., \& Ziegler, B.L. 2007, A\&A, 473, 761

Kronberger, T., Kapferer, W., Unterguggenberger, S., Schindler, S., \& Ziegler, B.L. 2008, A\&A, 483, 783

Mamon, G.A., Sanchis, T., Salvador-Solé, E., \& Solanes, J.M. 2004, A\&A, 414, 445

Matthews, L.D., \& Uson, J.M. 2008, AJ, 135, 291

Mei, S., Blakeslee, J.P., Côté, P., Tonry, J.L., West, M.J., Ferrarese, L., Jordán, A., Peng, E.W., Anthony, A., \& Merritt, D. 2007, ApJ, 655, 144

Peng, C.Y., Ho, L.C., Impey, C.D., \& Rix, H.W. 2002, AJ, 124, 266

Perley, R.A., \& Taylor, G.B. 2003, The VLA Calibrator Manual

Sanchis, T., Solanes, J.M., Salvador-Solé, E., Fouqué, P., \& Manrique, A. 2002, ApJ, 580, 164

Sanchis, T., Mamon, G.A., Salvador-Solé, E., \& Solanes, J.M. 2004, A\&A, 418, 393 
Solanes, J.M., Manrique, A., González-Casado, G., García-Gómez, C., Giovanelli, R., \& Haynes, M.P. 2001, ApJ, 548, 97

Solanes, J.M., Sanchis, T., Salvador-Solé, E., Giovanelli, R., \& Haynes, M.P. 2002, AJ, 124, 2440

Tully, R.B., \& Shaya, E.J. 1984, ApJ, 281, 31

Uson, J.M., \& Cotton, W.D. 2008, A\&A, 486, 647

Uson, J.M., \& Matthews, L.D. 2003, AJ, 125, 2455

Valenzuela, O., Rhee, G., Klypin, A., Governato, F., Stinson, G., Quinn, T., \& Wadsley, J. 2007, ApJ, 657, 773

Verheijen, M.A.W., \& Sancisi, R. 2001, A\&A, 370, 765

Vollmer, B., Cayatte, V., Balkowski, C., \& Duschl, W.J. 2001, ApJ, 561, 708

Warmels, R.H. 1986, Ph.D. thesis, University of Groningen

Yasuda, N., Fukugita, M., \& Okamura, S. 1997, ApJS, 108, 417 (YFO97) 


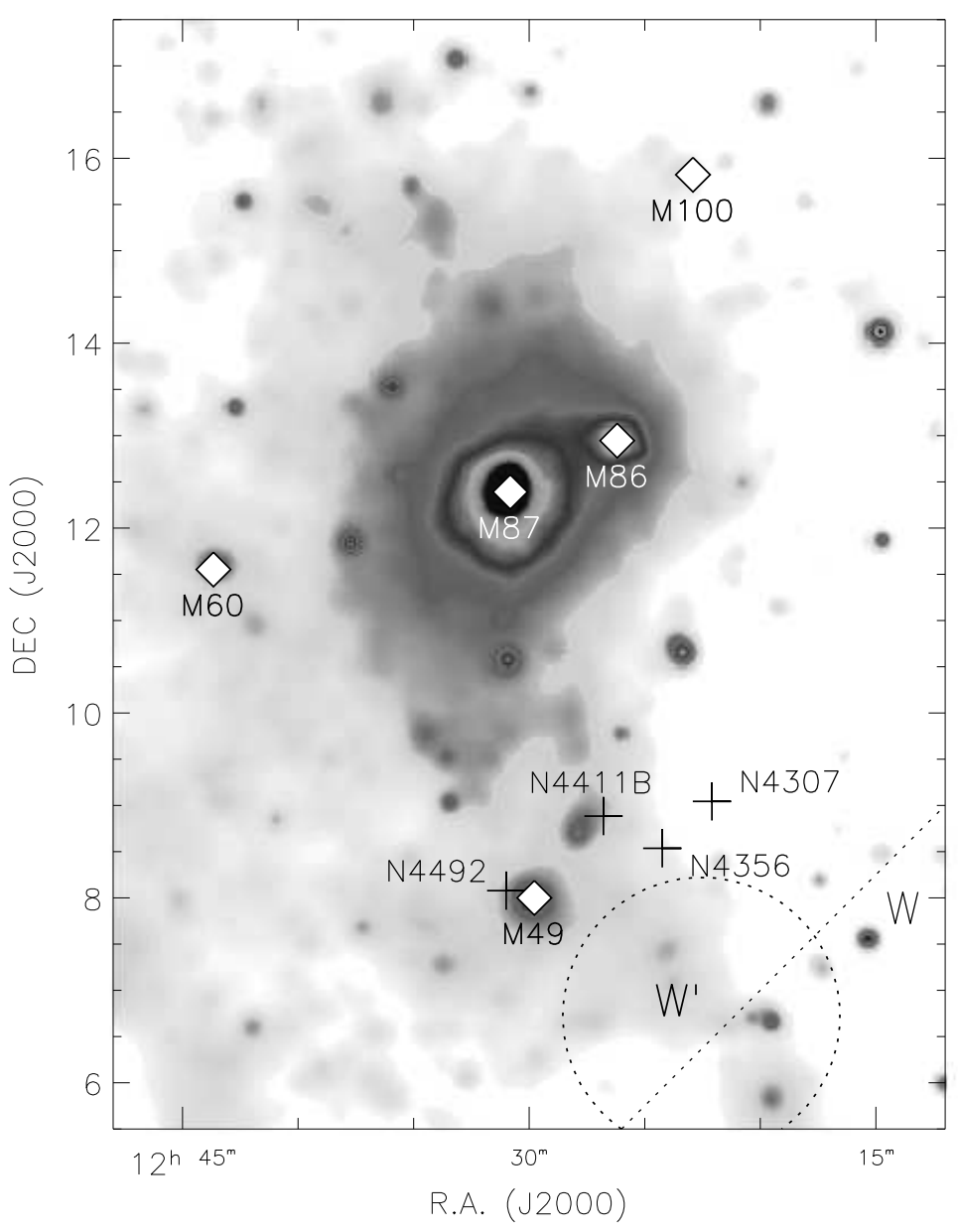

Fig. 1. - Sky distribution of our 4 main targets. The positions of five dominant Virgo cluster galaxies are marked by open diamonds (top to bottom: M100, M86, M87, M60, and M49). Overlaid on the figure it is the greyscale map of the X-ray emission in the cluster from the ROSAT All-Sky Survey in the 0.4-2.4 keV band (Böhringer et al. 1994). The location of W and W' clouds is also shown. 


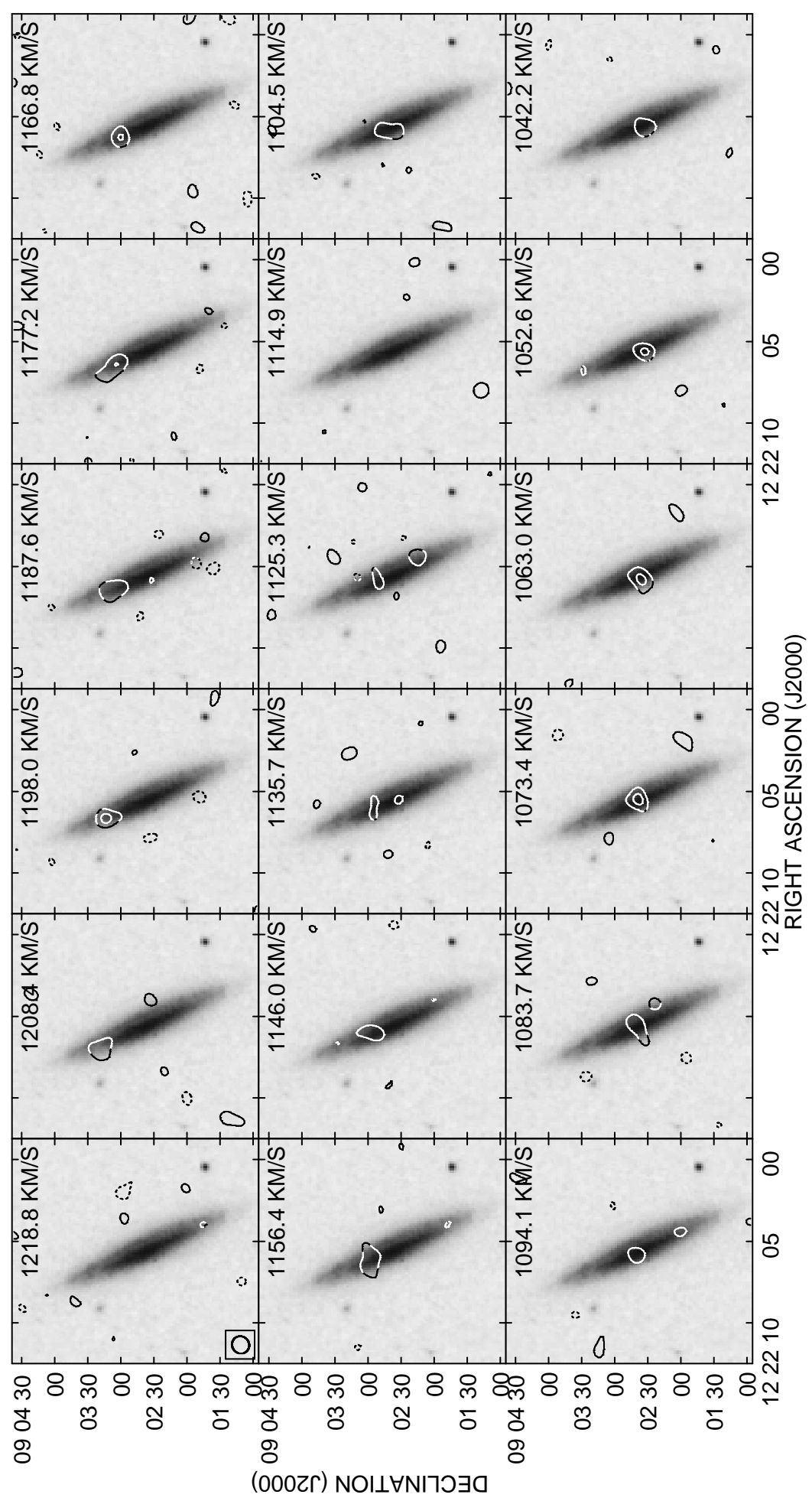

Fig. 2.- H I channel images for NGC 4307 overlaid on the DSS image of the galaxy. The maps were obtained by averaging the channels in pairs. Only channels containing line emission are plotted, bracketed by two noise channels. The heliocentric velocity of each panel is given in the upper right corner. The contours are drawn at ( -2 [absent], -1 (dashed), 1, 2) $\times 3 \sigma$, where $\sigma \sim 0.42 \mathrm{mJy}_{\text {beam }^{-1}}$ is the rms noise level (see Table 3). 


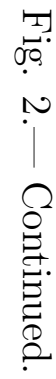

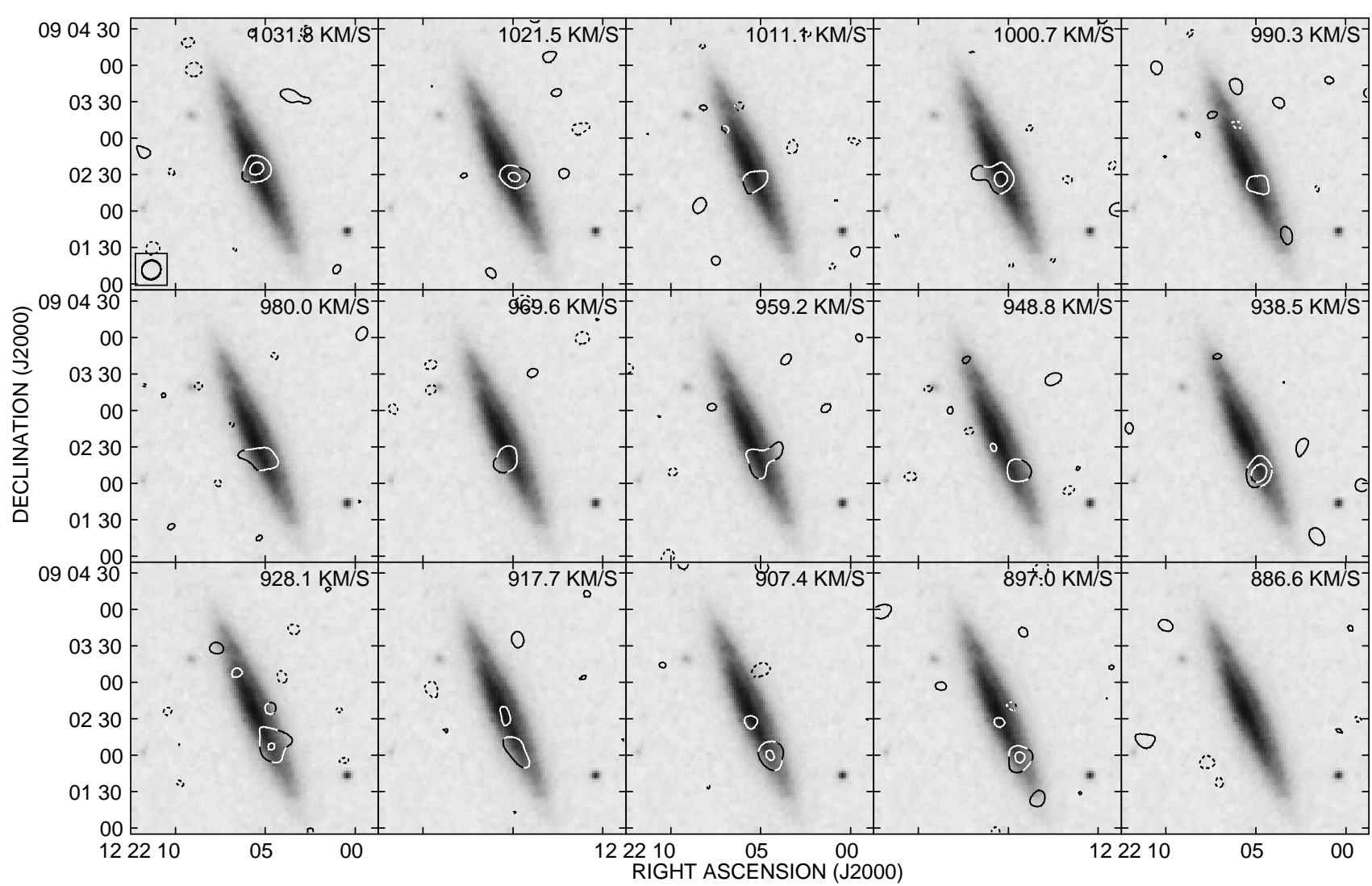


Fig. 3.- H I synthesis results for NGC 4307. (a) H I column density contours overlaid on the optical image of the galaxy obtained from the DSS. The contours levels are ( -2 [absent], -1 (dashed), 1, 2, 4, 8) $\times 31 \mathrm{mJy}$ beam ${ }^{-1} \mathrm{~km} \mathrm{~s}^{-1}$. The size of the synthesized beam is plotted in the lower left corner. (b) Linear gray-scale map of the H I column density. The grayscale range is 31-276 mJy beam ${ }^{-1} \mathrm{~km} \mathrm{~s}^{-1}$. (c) Velocity field of the H I with contours and grayscales ranging from $899 \mathrm{~km} \mathrm{~s}^{-1}$ (light gray) to $1261 \mathrm{~km} \mathrm{~s}^{-1}$ (dark gray) in increments of $20.8 \mathrm{~km} \mathrm{~s}^{-1}$. The adopted heliocentric systemic velocity is indicated by a thick black contour. (d) H I position-velocity plot along the major axis. The data have been averaged in velocity (joining the channels in pairs) and spatially along a $8^{\prime \prime}$-wide strip through the optical center of the galaxy with a position angle $\Gamma=25^{\circ}$ (East of North). Contour levels are at $(-\sqrt{2}$ [absent],-1 (dashed) $, 1, \sqrt{2}, 2) \times 1.44$ mJy beam $^{-1}$. The gray scale is linear in the range [0.96-3.11] mJy beam ${ }^{-1}$. A pair of vertical arrows are drawn to indicate the extent of the optical disk. (e) Integrated H I line profile from our VLA observations (solid line) and ALFALFA (dotted line). The vertical arrow indicates the systemic velocity from the VLA profile.
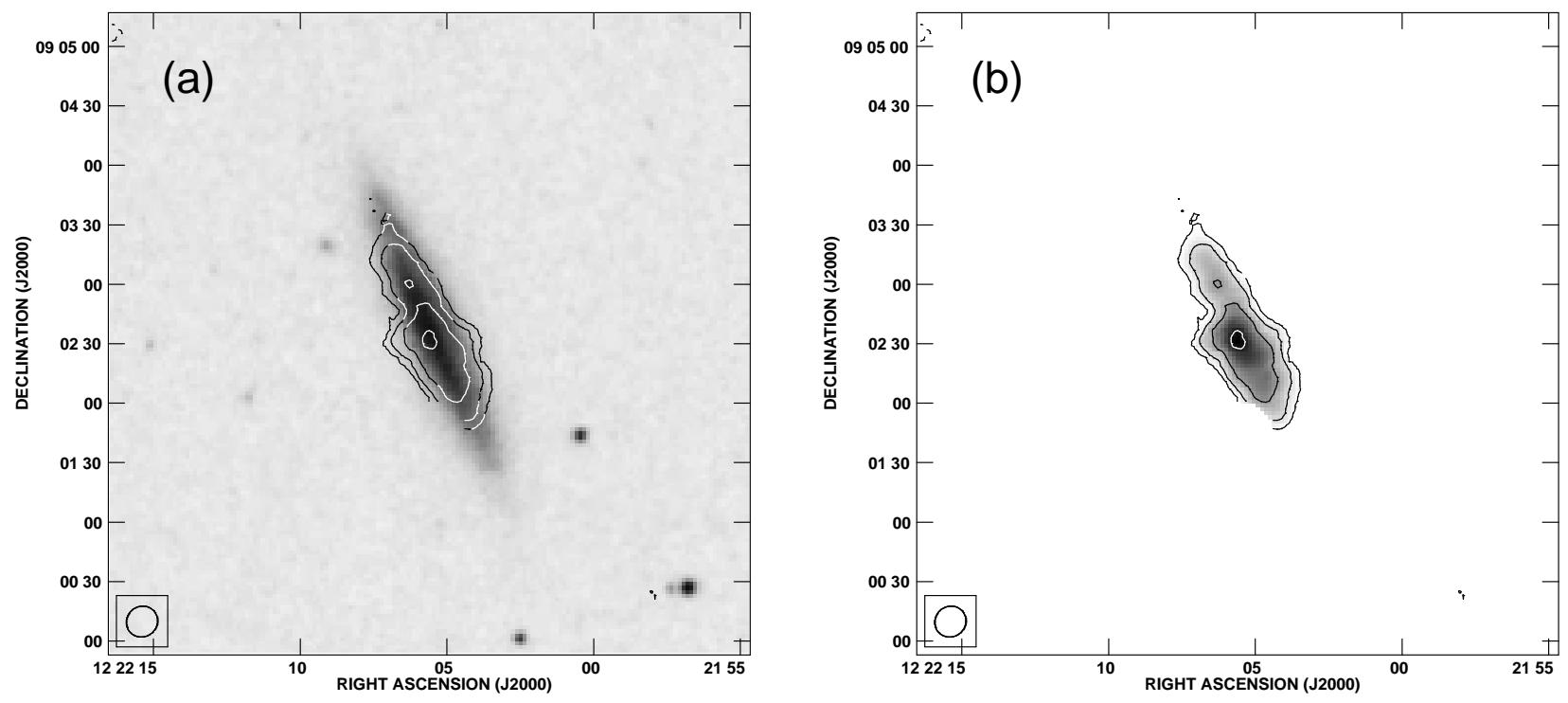

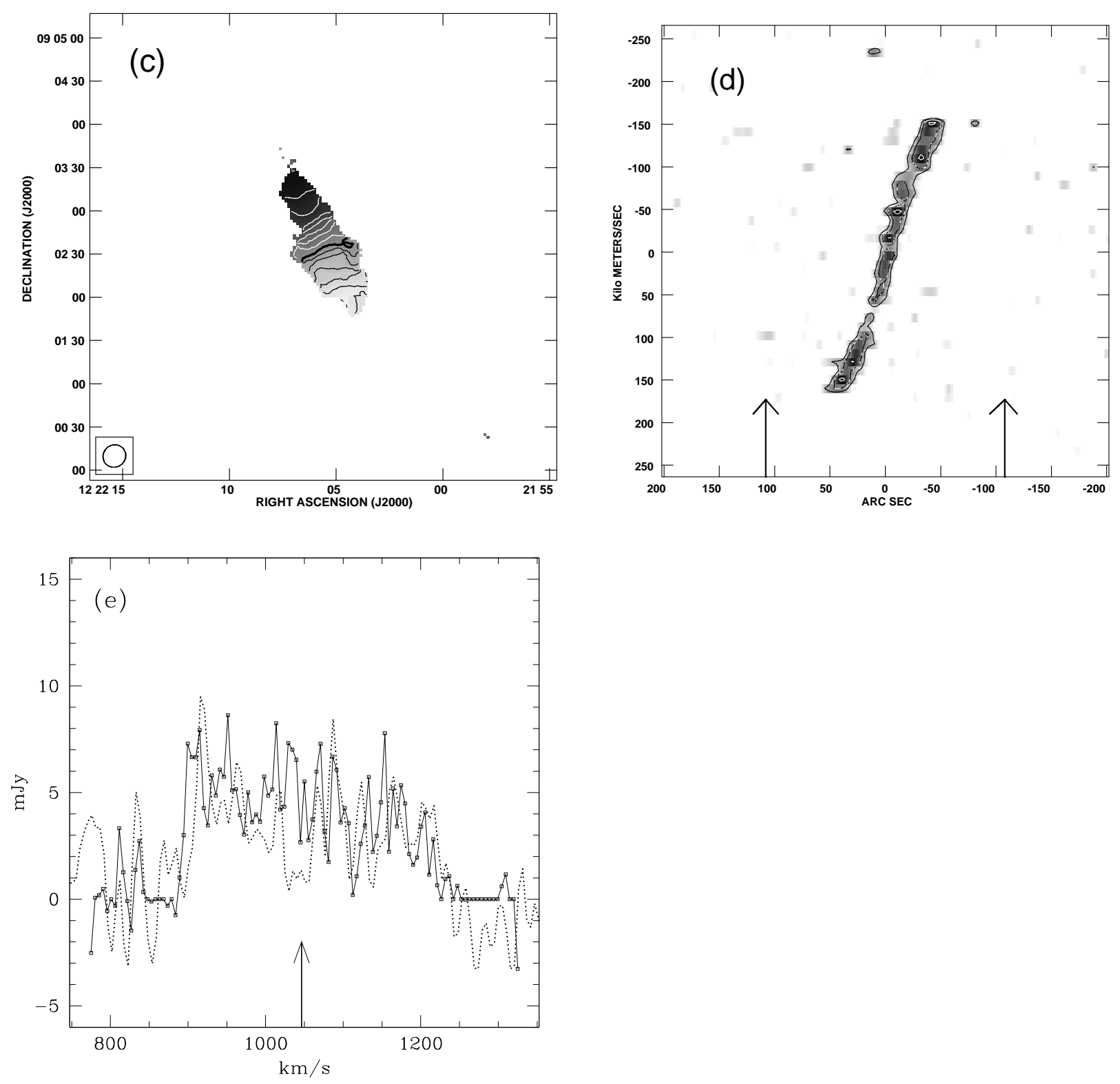

Fig. 3.- Continued. 

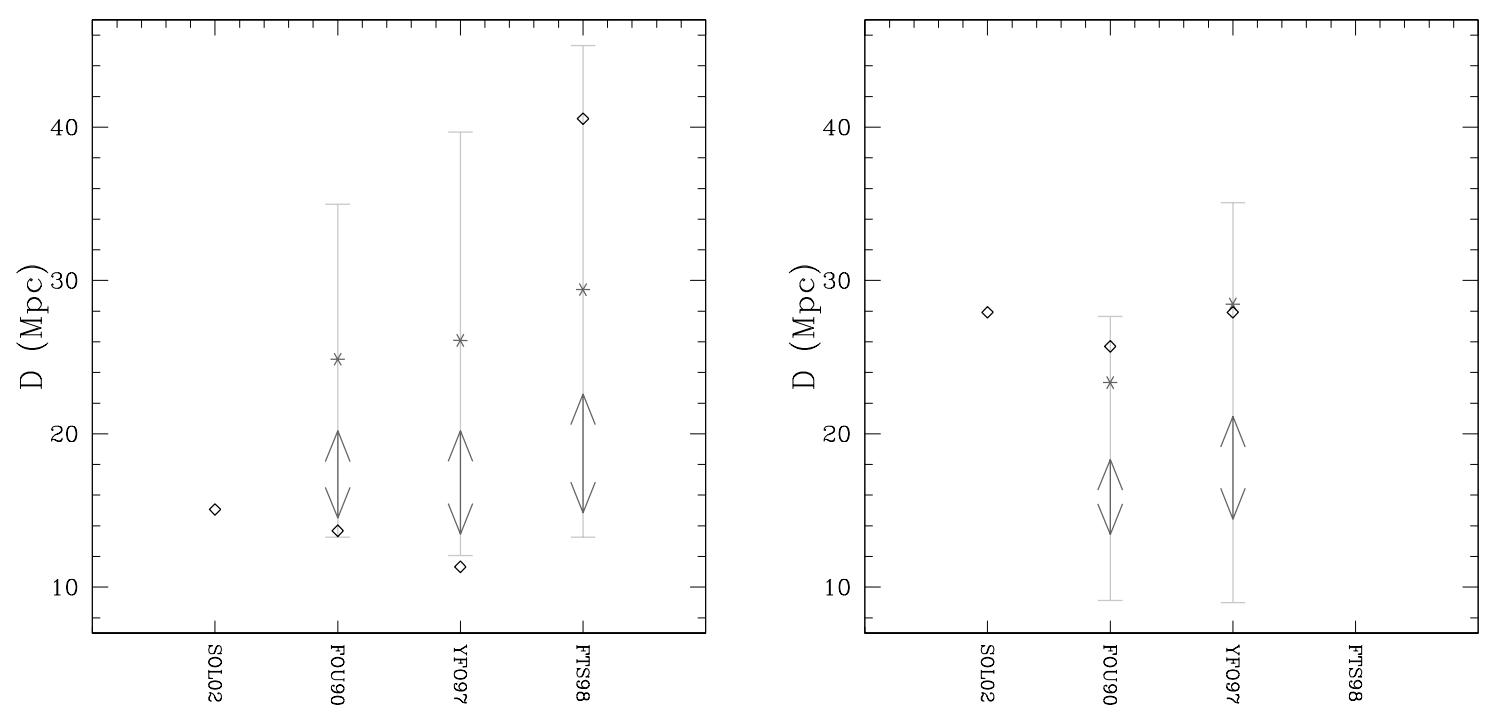

Fig. 4.- TF distances to the disk galaxies NGC 4411A (a) and NGC 4411B (b). The open diamonds indicate the radial distance to the galaxies published in several TF studies used in Sol02, quoted in the horizontal axis (see caption of Fig. 5). Vertical bars show the ranges of distances allowed by these studies when our VLA H I linewidth estimates and the most extreme values of the inclination ever inferred for each object are used. Double pointed arrows encompass the ranges of distances corresponding to the values of inclinations and errors derived from our H I data. Distance estimates resulting from the structural decomposition of the SDSS r-band images are indicated by asterisks. 


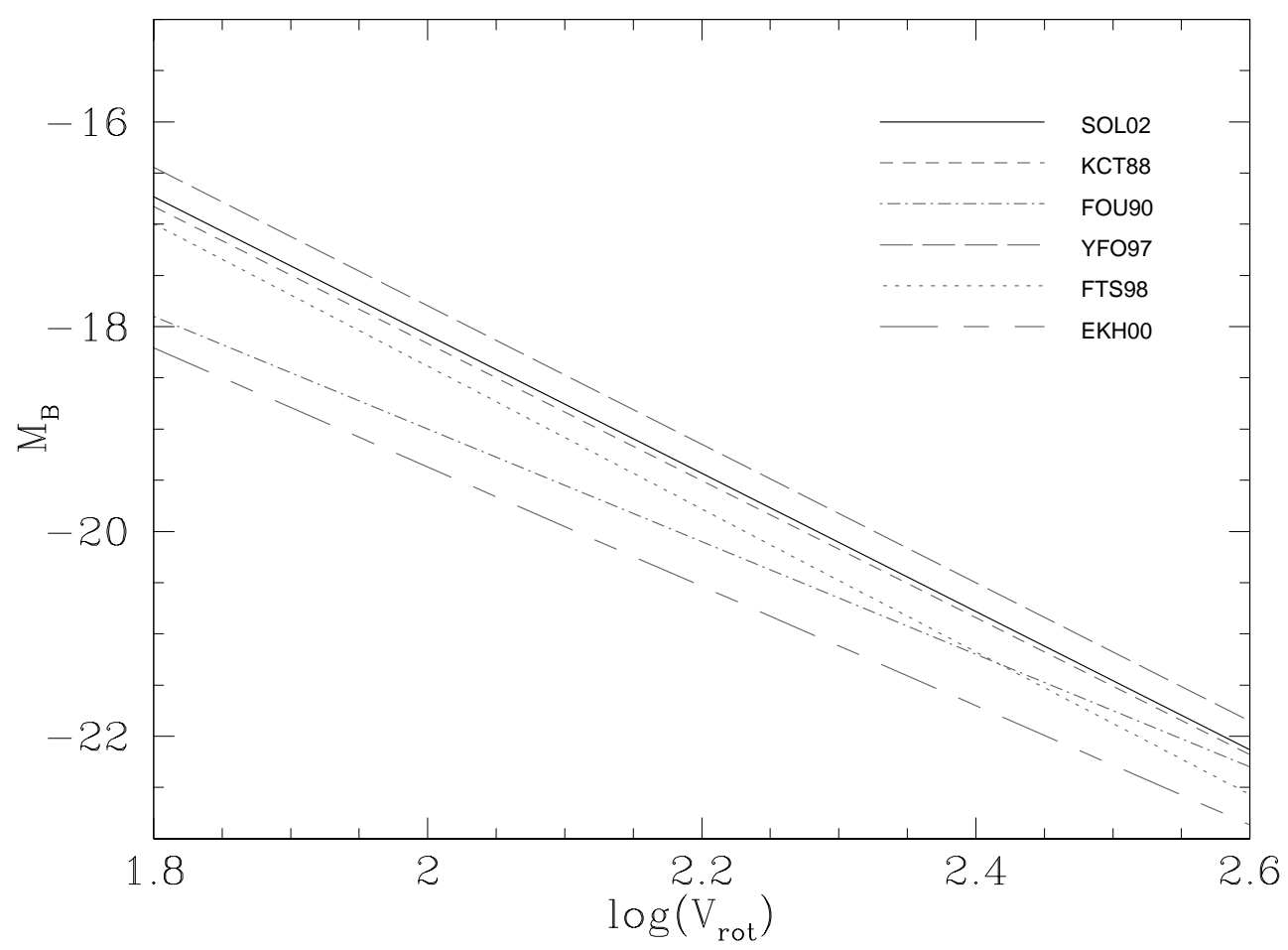

Fig. 5.- B-band TF templates considered in Sol02. The acronyms correspond to Yasuda, Fukugita, \& Okamura (1997) (YFO97), Kraan-Korteweg. Cameron, \& Tammann (1988) (KCT88), Fouqué et al. (1990) (Fou90), Federspiel, Tammann, \& Sandage (1998) (FTS98), and Ekholm et al. (2000) (Ekh00). The relation resulting from the homogenization of the eight TF catalogs used by Sol02 is also included. 
Table 1. Properties of the target galaxies

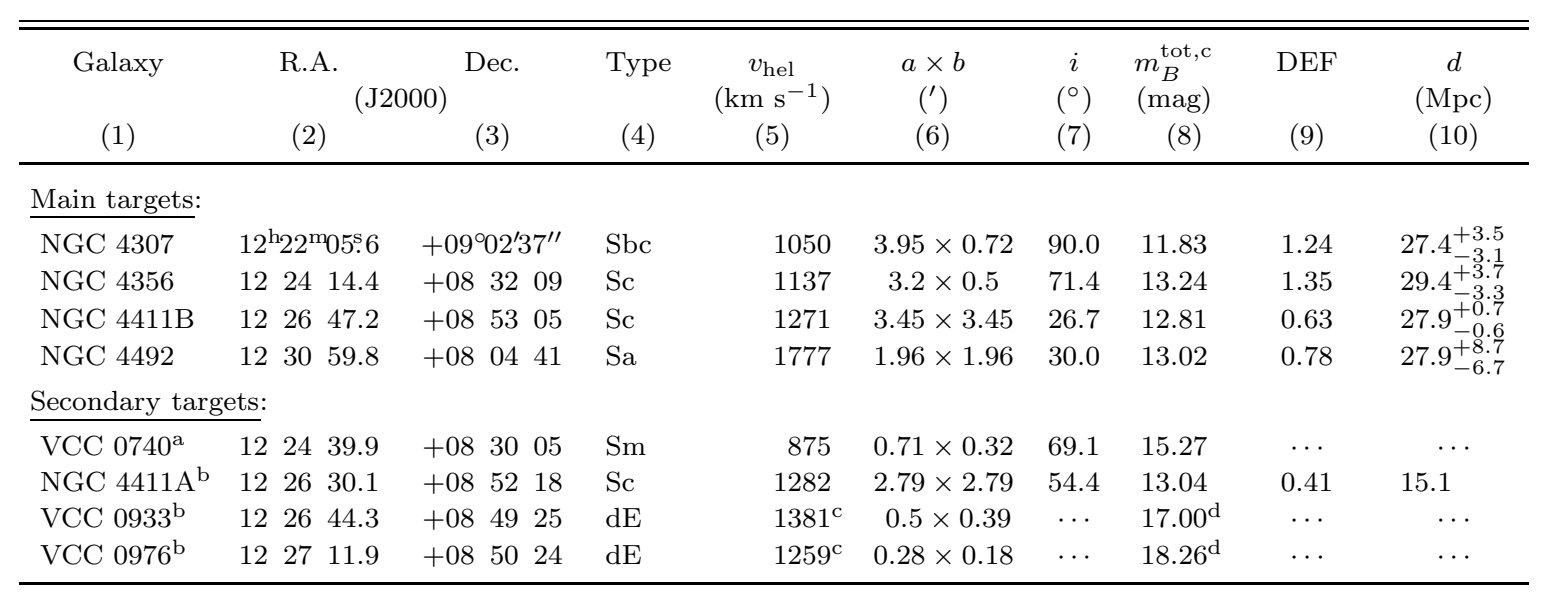

a: in the same field of NGC 4356

b: in the same field of NGC 4411B.

c: derived from optical measurements as listed in the Galaxy On-Line Database Milano Network (GOLDMine).

d: photographic magnitude.

Note. - Description of columns:

(1) NGC or VCC designations.

(2)-(3) J2000 position listed in the Lyon-Meudon Extragalactic Database (LEDA).

(4) Morphological type from GOLDMine.

(5) Heliocentric radial velocity obtained from $\mathrm{H}$ i profile measurements.

(6) Angular size of the semi-major and semi-minor optical axes determined at the surface brightness of $25 \mathrm{mag} \operatorname{arcsec}^{-2}$ from GOLDMine.

(7) Inclination between the line-of-sight (LOS) and polar axis of the optical image listed in the LEDA.

(8) Total apparent $B$ magnitude corrected from inclination and extinction effects as listed in the LEDA

(9) H I deficiency parameter calculated using the definition in equation (1) and the optical sizes and H I fluxes from conventional single-dish measurements attributed in the Arecibo General Catalog, a private compilation of $21 \mathrm{~cm}$ line measurements maintained by R. Giovanelli and M. P. Haynes at Cornell University.

(10) TF radial distance estimate from $\mathrm{Sol02}$ 
Table 2. Summary of the observations

\begin{tabular}{|c|c|c|c|c|}
\hline Field & NGC 4307 & NGC 4356 & NGC 4492 & NGC 4411B \\
\hline Array configuration & CS & CS & CS & CS \\
\hline Observing dates & 09-07-2005 & $16-07-2005$ & $\begin{array}{l}12-08-2005 \text { and } \\
18-09-2005\end{array}$ & $\begin{array}{l}01-10-2005 \text { and } \\
02-10-2005\end{array}$ \\
\hline Total time on source $(\mathrm{hr})$ & 6.23 & 6.12 & 6.25 & 6.3 \\
\hline Phase center, $\alpha(\mathrm{J} 2000)$ & $12^{\mathrm{h}} 22^{\mathrm{m}} 05^{\mathrm{s}} 6$ & $12^{\mathrm{h}} 24^{\mathrm{m}} 14^{\mathrm{s}} .5$ & $12^{\mathrm{h}} 30^{\mathrm{m}} 47^{\mathrm{s}} .7$ & $12^{\mathrm{h}} 26^{\mathrm{m}} 47^{\mathrm{s}} \cdot 2$ \\
\hline Phase center, $\delta(\mathrm{J} 2000)$ & $09^{\circ} 02^{\prime} 37^{\prime \prime}$ & $08^{\circ} 32^{\prime} 09^{\prime \prime}$ & $08^{\circ} 04^{\prime} 41^{\prime \prime}$ & $08^{\circ} 53^{\prime} 04^{\prime \prime}$ \\
\hline Flux calibrator (J2000) & $1331+305$ & $1331+305$ & $1331+305$ & $1331+305$ \\
\hline Phase calibrator (J2000) & $1229+020$ & $1229+020$ & $1229+020$ & $1254+116$ \\
\hline Bandwidth (1st/2nd IF pair, MHz) & $1.562 / 1.562$ & $1.562 / 1.562$ & $1.562 / 3.125$ & $1.562 / 3.125$ \\
\hline Number of channels (1st/2nd IF pair) & $63 / 63$ & $63 / 63$ & $63 / 31$ & $63 / 31$ \\
\hline Channel width $^{\mathrm{a}}$ (1st/2nd IF pair, kHz) & $24.4 / 24.4$ & $24.4 / 24.4$ & $24.4 / 97.6$ & $24.4 / 97.6$ \\
\hline $\begin{array}{l}\text { Central heliocentric velocity } \\
\left.\text { (1st/2nd IF pair, } \mathrm{km} \mathrm{s}^{-1}\right)\end{array}$ & $920 / 1180$ & $940 / 1180$ & $1777 / 1777$ & $1309 / 1309$ \\
\hline
\end{tabular}

a: after Hanning smoothing.

b: optical definition. 
Table 3. Characteristics of the deconvolved images

\begin{tabular}{|c|c|c|c|c|}
\hline Field & NGC 4307 & NGC 4356 & NGC $4492^{\mathrm{b}}$ & NGC $4411 B$ \\
\hline $\begin{array}{l}\text { Robustness parameter } \\
\text { Gaussian taper width at } 30 \% \text { level }(\mathrm{k} \lambda) \\
\text { Synthesized beam FWHM }\left(^{\prime \prime}\right) \\
\text { Synthesized beam position angle }\left(^{\circ}\right) \\
\text { rms noise per channel in line-free channels }\left(\mathrm{mJy} \mathrm{beam}^{-1}\right) \\
1 \sigma \text { limiting column density per channel }\left(10^{19} \mathrm{~cm}^{-2}\right) \\
\left.\text { rms noise in total intensity map (mJy beam }{ }^{-1} \mathrm{~km} \mathrm{~s}^{-1}\right) \\
1 \sigma \text { limiting column density in total intensity map } \\
\left(10^{19} \mathrm{~cm}^{-2} \mathrm{~km} \mathrm{~s}^{-1}\right)\end{array}$ & $\begin{array}{c}0.7 \\
0 \\
16.09 \times 15.26 \\
-51.1 \\
\sim 0.57^{\mathrm{a}} \\
1.32 \\
10.3 \\
\\
4.6\end{array}$ & $\begin{array}{c}\quad 0.7 \\
0 \\
16.29 \times 15.47 \\
-55.7 \\
\sim 0.60^{\mathrm{a}} \\
1.36 \\
11.1 \\
\\
\quad 4.8\end{array}$ & $\begin{array}{c}1 \\
9 \\
23.03 \times 21.18 \\
-55.5 \\
0.35 \\
1.65 \\
16.9 \\
\\
3.7\end{array}$ & $\begin{aligned} & 0.7 \\
& 0 \\
16.74 & \times 14.11 \\
- & 38.8 \\
\sim & 0.60 \\
& 1.46 \\
& 9.0 \\
& \\
& 4.2\end{aligned}$ \\
\hline
\end{tabular}

a: $\sim 0.42$ for the cube obtained by averaging each two channels.

b: corresponding to images obtained from IF2 (see Table 3 ). 
Table 4. Results from our VLA data and recent Arecibo measurements

\begin{tabular}{|c|c|c|c|c|c|c|c|c|c|c|}
\hline \multirow[b]{2}{*}{$\begin{array}{c}\text { Galaxy } \\
\text { (1) }\end{array}$} & \multirow[b]{2}{*}{$\begin{array}{c}W_{20} \\
\left(\mathrm{~km} \mathrm{~s}^{-1}\right) \\
(2)\end{array}$} & \multirow[b]{2}{*}{$\begin{array}{c}W_{50} \\
\left(\mathrm{~km} \mathrm{~s}^{-1}\right) \\
(3)\end{array}$} & \multicolumn{2}{|l|}{ VLA } & \multirow[b]{2}{*}{$\begin{array}{c}v_{\text {sys }} \\
\left(\mathrm{km} \mathrm{s}^{-1}\right) \\
(6)\end{array}$} & \multirow[b]{2}{*}{$\begin{array}{c}D_{\mathrm{HI}} \\
\left({ }^{\prime}\right) \\
(7)\end{array}$} & \multirow[b]{2}{*}{$\begin{array}{c}W_{50} \\
\left(\mathrm{~km} \mathrm{~s}^{-1}\right) \\
(8)\end{array}$} & \multicolumn{2}{|c|}{ ALFALFA } & \multirow[b]{2}{*}{$\begin{array}{c}v_{\text {sys }} \\
\left(\mathrm{km} \mathrm{s}^{-1}\right) \\
(11)\end{array}$} \\
\hline & & & $\begin{array}{c}\int S d v \\
\left(\mathrm{Jy} \mathrm{km} \mathrm{s}^{-1}\right) \\
(4)\end{array}$ & $\begin{array}{c}\text { DEF } \\
(5)\end{array}$ & & & & $\begin{array}{c}\int S d v \\
\left(\mathrm{Jy} \mathrm{km} \mathrm{s}^{-1}\right) \\
(9)\end{array}$ & $\begin{array}{l}\text { DEF } \\
(10)\end{array}$ & \\
\hline NGC 4307 & $327 \pm 8$ & $286 \pm 15$ & $1.48 \pm 0.09$ & 1.32 & $1048 \pm 4$ & $1.83 \pm 0.16$ & $314 \pm 41$ & $1.21 \pm 0.10$ & 1.41 & $1065 \pm 21$ \\
\hline NGC 4356 & $204 \pm 7$ & $154 \pm 27$ & $0.44 \pm 0.04$ & 1.71 & $1120 \pm 6$ & $1.41 \pm 0.14$ & $237 \pm 25$ & $0.87 \pm 0.08$ & 1.41 & $1092 \pm 12$ \\
\hline NGC 4411B & $93 \pm 1$ & $77 \pm 1$ & $18.91 \pm 0.95$ & 0.13 & $1270 \pm 0$ & $4.03 \pm 0.29$ & $82 \pm 2$ & $17.57 \pm 0.07$ & 0.16 & $1272 \pm 1$ \\
\hline NGC $4492^{\mathrm{a}}$ & $141 \pm 12$ & $79 \pm 25$ & $0.43 \pm 0.04$ & 1.21 & $1768 \pm 7$ & $1.38 \pm 0.17$ & $182 \pm 9$ & $0.59 \pm 0.07$ & 1.07 & $1740 \pm 5$ \\
\hline VCC $740^{\mathrm{b}}$ & $132 \pm 3$ & $109 \pm 5$ & $1.54 \pm 0.09$ & -0.21 & $875 \pm 1$ & $1.47 \pm 0.14$ & $97 \pm 5$ & $1.39 \pm 0.06$ & -0.17 & $877 \pm 2$ \\
\hline NGC $4411 \mathrm{~A}^{\mathrm{c}}$ & $107 \pm 1$ & $90 \pm 1$ & $12.47 \pm 0.63$ & -0.03 & $1278 \pm 0$ & $3.84 \pm 0.27$ & $89 \pm 0$ & $13.6 \pm 0.07$ & -0.07 & $1278 \pm 0$ \\
\hline
\end{tabular}

a: Columns (2)-(6) show measurements from IF1 integrated profile (resolution $\sim 5.2 \mathrm{~km} \mathrm{~s}^{-1}$ ), whereas $(7)$ was measured on IF2 images.

b: in the same field of NGC 4356 .

c: in the same field of NGC 4411B.

Note. - Column description:

(1) Target name.

(2)-(3) Width of the $\mathrm{H}$ I line profile measured at $20 \%$ and $50 \%$ of the peak flux, respectively. Adopted broadening corrections correspond to a spectral resolution of $5.2 \mathrm{~km} \mathrm{~s}^{-1}$ (see text).

(4) Integrated total flux observed with the VLA.

(5) Corresponding value of the H I deficiency parameter recalculated from our VLA measurements.

(6) Heliocentric systemic velocity.

(7) $\mathrm{H}$ i diameter measured at a column density of $10^{20}$ atoms $\mathrm{cm}^{-2}$ (no correction for beam smearing is applied).

(8) Width of the $\mathrm{H}$ i line profile at $50 \%$ of the peak flux measured by ALFALFA.

(9) Integrated total flux measured by ALFALFA.

(10) Corresponding value of the $\mathrm{H}_{\mathrm{I}}$ deficiency parameter recalculated from ALFALFA measurements.

(11) Heliocentric systemic velocity measured by ALFALFA. 